\title{
Disruption of Cancer Cell Replication by Alternating Electric Fields
}

\author{
Eilon D. Kirson, ${ }^{1}$ Zoya Gurvich, ${ }^{2}$ Rosa Schneiderman, ${ }^{2}$ Erez Dekel, ${ }^{3}$ Aviran Itzhaki, ${ }^{4}$ Yoram Wasserman,,${ }^{1,4}$ \\ Rachel Schatzberger, ${ }^{2}$ and Yoram Palti ${ }^{2}$ \\ ${ }^{1}$ Department of Biomedical Engineering, NovoCure Ltd., Haifa, Israel; ${ }^{2}$ B. Rappaport Faculty of Medicine, Technion-Israel Institute of Technology, Haifa, Israel; ${ }^{3}$ Department \\ of Molecular Cell Biology, Weizmann Institute of Science, Rehovot, Israel; and ${ }^{4}$ Elisha Medical Centre, Haifa, Israel
}

\begin{abstract}
Low-intensity, intermediate-frequency $(100-300 \mathrm{kHz})$, alternating electric fields, delivered by means of insulated electrodes, were found to have a profound inhibitory effect on the growth rate of a variety of human and rodent tumor cell lines (Patricia C, U-118, U-87, H-1299, MDA231, PC3, B16F1, F-98, C-6, RG2, and CT-26) and malignant tumors in animals. This effect, shown to be nonthermal, selectively affects dividing cells while quiescent cells are left intact. These fields act in two modes: arrest of cell proliferation and destruction of cells while undergoing division. Both effects are demonstrated when such fields are applied for $24 \mathrm{~h}$ to cells undergoing mitosis that is oriented roughly along the field direction. The first mode of action is manifested by interference with the proper formation of the mitotic spindle, whereas the second results in rapid disintegration of the dividing cells. Both effects, which are frequency dependent, are consistent with the computed directional forces exerted by these specific fields on charges and dipoles within the dividing cells. In vivo treatment of tumors in C57BL/6 and BALB/c mice (B16F1 and CT-26 syngeneic tumor models, respectively), resulted in significant slowing of tumor growth and extensive destruction of tumor cells within 3-6 days. These findings demonstrate the potential applicability of the described electric fields as a novel therapeutic modality for malignant tumors.
\end{abstract}

\section{INTRODUCTION}

In the laboratory setting and in clinical practice, alternating electric fields show a wide range of effects on living tissues. At very low frequencies (under $1 \mathrm{kHz}$ ), alternating electric fields stimulate excitable tissues through membrane depolarization (1). The transmission of such fields by radiation is insignificant, and therefore they are usually applied directly by contact electrodes, although some applications have also used insulated electrodes. Some well-known examples of such effects include nerve, muscle, and heart stimulation by alternating electric fields $(1,2)$. In addition, low-frequency pulsed electric fields have been claimed to stimulate bone growth and accelerate fracture healing (3). However, as the frequency of the electric field increases above $1 \mathrm{kHz}$, the stimulatory effect diminishes. Under these conditions, although a greater fraction of the fields penetrates the cells, due to the parallel resistor-capacitor nature of all biological membranes, the stimulatory power greatly diminishes as the alternating cell membrane hyper-depolarization cycles are integrated such that the net effect is nulled. At very high frequencies (i.e., above many $\mathrm{MHz}$ ), although the integration becomes even more effective, a completely different biological effect is observed. At these frequencies tissue heating becomes dominant due to dielectric losses. This effect becomes more intense as frequency, field intensity, or tissue dissipation factor increases (4). This phenomenon serves as the basis for some commonly used medical treatment modalities including diathermy and radio frequency tumor ablation, which can be applied through insulated electrodes (5). Intermediate-frequency electric

\footnotetext{
Received 1/11/04; revised 2/12/04; accepted 2/17/04.

Grant support: NovoCure Ltd.

The costs of publication of this article were defrayed in part by the payment of page charges. This article must therefore be hereby marked advertisement in accordance with 18 U.S.C. Section 1734 solely to indicate this fact.

Requests for reprints: Yoram Palti, Department of Physiology, B. Rappaport Faculty of Medicine, Technion-Israel Institute of Technology, Haifa 31906, Israel. Phone: 972-4-8501204; Fax: 972-4-8501207; E-mail: yoramp@netvision.net.il.
}

fields (i.e., tens of kilohertz to megahertz) alternate too fast for causing nerve-muscle stimulation and involve only minute dielectric losses (heating). Such fields of low to moderate intensities are commonly considered to have no biological effect (4). However, a number of nonthermal effects of minor biological consequence have been reported even at low field intensities. These include microscopic particle alignment (i.e., the pearl chain effect; Ref. 6) and cell rotation $(7,8)$. With pulsed electric fields of $10^{3} \mathrm{~V} / \mathrm{cm}$ and $100-\mathrm{ms}$ pulse length, reversible pore formation appears in the cell membrane, a phenomenon usually called electroporation (9).

In the present study we show for the first time, to our knowledge, that very low-intensity $(<2 \mathrm{~V} / \mathrm{cm})$, intermediate-frequency $(100-300$ $\mathrm{kHz}$ ), alternating electric fields induced by insulated electrodes have specific inhibitory effects on dividing cells in culture. We demonstrate that applying these fields to cancerous cells leads to proliferation arrest and cell destruction. When applied to syngeneic mice tumor models, these tumor treating fields (TTFields) cause a significant reduction in tumor growth rate without any significant side effects.

\section{MATERIALS AND METHODS}

In Vitro Experimental Set Up. Cultures were grown in standard culture dishes (4-well cell culture chambers; SN 138121; Nalge Nunc International). The TTFields were generated by pairs of $15-\mathrm{mm}$-long, completely insulated wires (P/N K-30-1000; VT Corporation; outer diameter, $0.5 \mathrm{~mm}$; ethylene tetrafluoroethylene insulation thickness, $0.125 \mathrm{~mm}$; dielectric breakdown, $1800 \mathrm{~V} / \mathrm{mil}$ ) fixed to the bottom of each dish at a distance of $1 \mathrm{~mm}$ from each other. The wires were connected to an oscillator (GFG8219A; Instek) and a high-voltage amplifier (A303; A. A. Lab Systems Ltd.) that generated the required sine-wave signals (range, $300-800 \mathrm{~V}$ ). Cells were plated by carefully smearing $10 \mu \mathrm{l}$ of DMEM (Biological Industries Ltd., Beit Haemek, Israel) containing $1.5 \times 10^{4}$ cells along the gap between the wires (Fig. $1 A$ ). After the cells settled and attached to the plate surface, $500 \mu \mathrm{l}$ of DMEM were added to each culture dish, which was then transferred to a $5 \% \mathrm{CO}_{2}$ humidified incubator held at $36^{\circ} \mathrm{C}$. The culture was incubated for a control period of $24 \mathrm{~h}$ before treatment. Culture medium was replaced manually every $24 \mathrm{~h}$ throughout the experiments. TTFields were then applied by connecting the wires to a high-voltage amplifier operated by a signal generator with frequency and amplitude controls. Finite element simulation of the TTFields generated between the wires demonstrated that the field in the vicinity of the cell culture was homogenous (not shown). Eleven different types of cancerous cell lines were subjected to TTFields. These included human melanoma (Patricia), glioma (U-118, U-87), Lung (H-1299), prostate (PC3), and breast (MDA231) cancerous cell lines as well as mouse melanoma (B16F1), rat glioma (F-98, C-6, and RG2), and mouse adenocarcinoma (CT-26) cell lines (all from American Type Culture Collection, except for Patricia, which was a generous gift from Dr. Ruth Halaban, Department of Dermatology, Yale University School of Medicine). In addition, a noncancerous cell line (BHK) was grown under conditions that stunt cell replication $(0.1 \%$ FCS $)$ and then subjected to TTFields. Also, segments of excised rat mesentery and diaphragm were subjected to the fields in vitro. Colorimetric cell counts were made every $24 \mathrm{~h}$ after seeding using the standard 2,3-bis(2-methoxy-4-nitro-5-sulfophenyl)-5[(phenylamino)carbonyl]-2H-tetrazolium hydroxide method to measure cell proliferation as described previously (10) using cell proliferation assay kit (Biological Industries, Beit Haemek, Israel). In brief, culture media was replaced with $0.2 \mathrm{ml}$ of preheated 2,3-bis(2-methoxy-4-nitro-5-sulfophenyl)5-[(phenylamino)carbonyl]-2H-tetrazolium hydroxide reagent and incubated for $1 \mathrm{~h}$ at $37^{\circ} \mathrm{C}$ in a $5 \% \mathrm{CO}_{2}$ incubator. After incubation and gentle stirring, 
$0.15 \mathrm{ml}$ of the reaction solution was transferred to a 96-well plate (SN 92696; TPP, Trasandigen, Switzerland). The absorbance of the samples was then read with a spectrophotometer (Tecan ELISA Reader; $450 \mathrm{~nm}$ ). The colorimetric measurements at each time point were normalized to the measurement performed immediately before beginning of treatment. To verify that the colorimetric assessments were accurate, direct visual cell counts were performed on sample culture dishes. At the optic densities used (0.2-2), optic density was linearly related to the number of cells in the culture dishes $\left(n=10 ; r^{2}=0.99\right)$. The growth rate of both treated $\left(\mathrm{GR}_{\mathrm{t}}\right)$ and control cultures $\left(\mathrm{GR}_{\mathrm{c}}\right)$ was calculated for each experiment by plotting the optic density values on a logarithmic scale and fitting a linear regression line to the values. The growth rate for each culture dish was the slope of this linear regression. The therapeutic enhancement ratio (TER) was calculated as the ratio of the decrease in the growth rate of treated cells compared with the growth rate of control cells $\left[\left(\mathrm{GR}_{\mathrm{c}}-\mathrm{GR}_{\mathrm{t}}\right) /\right.$ $\mathrm{GR}_{\mathrm{c}}$ ]. Thus, if the increase in the number of treated cells is equal to that of the controls, TER $=0$; if the increase in cell number is smaller in the treated cultures than in the controls, TER $>0$; and if the number of cells in the treated cultures decreases absolutely, TER $>1$.

In time-lapse microphotography experiments, cell lines were grown on a 35-mm standard culture dish ( $\mathrm{SN} 430165$; Corning Inc.) by plating $3 \times 10^{4}$ cells in $2.5 \mathrm{ml}$ of DMEM with $25 \mathrm{mM}$ HEPES. The Petri dish temperature was controlled at $34^{\circ} \mathrm{C}(\mathrm{B} 16 \mathrm{~F} 1)$ or at $37^{\circ} \mathrm{C}$ (all other cell lines). Subsequently, two parallel insulated wires were positioned on the bottom of the dish with $1 \mathrm{~mm}$ distance between through which TTFields were applied. The entire set-up was placed on an inverted microscope (Eclipse TS-100; Nikon) and video microphotographs at $\times 200$ magnification were taken with a standard VCR camera (Handicam X 320; Sony). Photographs were captured using a personal computer every $60-120 \mathrm{~s}$ for $6-10 \mathrm{~h} /$ culture.

Fluorescent Labeling of $\boldsymbol{\alpha}$-Tubulin, Actin, and DNA. Mouse melanoma cells were grown on coverslips and subjected to TTFields for $24 \mathrm{~h}$. After treatment, the medium was removed, and the cells were washed in a buffer solution [10 mM 4-morpholineethanesulfonic acid, $150 \mathrm{~mm} \mathrm{NaCl}, 5 \mathrm{~mm}$ EGTA, $5 \mathrm{~mm} \mathrm{MgCl}_{2}$, and $5 \mathrm{~mm}$ glucose (pH 6.1)], permeabilized, and fixed with $0.5 \%$ Triton X-100 and $0.25 \%$ glutaraldehyde (Sigma) for $5 \mathrm{~min}$ and then post-fixed with $1 \%$ glutaraldehyde for $20 \mathrm{~min}$. Subsequently, the cells were washed in PBS and 1 mm sodium borohydride (Sigma) to eliminate autofluorescence. The coverslips were then incubated with a primary antibody clone for $\alpha$-tubulin (DM1A; Sigma) for $30 \mathrm{~min}$, washed, and incubated for 30 min with a secondary antibody (Alexa Fluor 488 goat antimouse IgG; Molecular Probes). Rhodamine-conjugated phalloidin (Sigma) was added with the secondary antibody to stain actin filaments. The cells were then washed and incubated with 4',6-diamidino-2-phenylindole (Molecular Probes) to stain the DNA. After staining, the coverslips were mounted and viewed with a fluorescence microscope at $\times 630$ magnification and photographed.

Electric Field Measurement. The electric field intensity in the culture medium was measured by means of a probe, consisting of two $(0.25 \mathrm{~mm}$ in diameter) insulated wires with exposed tips $0.5 \mathrm{~mm}$ apart, that was dipped in the culture medium. The wires were connected to a high-input impedance differential amplifier that translated the waveform amplitude into a calibrated steady voltage that was digitally recorded. Field intensities throughout the manuscript are expressed in peak voltage amplitude per centimeter $(\mathrm{V} / \mathrm{cm})$. Care was taken to eliminate any pickup from the field outside the culture medium. Continuous field monitoring could also be made by measuring the potential drop across a $100 \Omega$ resistor placed in series with one of the fieldgenerating wires. The voltage drop on this resistor was linearly correlated to the field intensity $\left(r^{2}=0.96\right)$. To verify that the experimental setups were not exposed to any significant magnetic fields, the electromagnetic radiation in the immediate vicinity of the treated cultures was measured using a loop antennae (EMCO $65071 \mathrm{kHz}$ to $30 \mathrm{MHz}$ ) connected to a spectrum analyzer (Anritsu 9 $\mathrm{kHz}$ to $2.2 \mathrm{GHz}$ ). The electromagnetic radiation in the $100-300-\mathrm{kHz}$ range within the incubators containing treated culture dishes was found to be $10^{-12}$ Tesla and within animal cages containing TTField-treated mice, $10^{-14}$ Tesla, i.e., negligible.

Finite Element Simulations of Electric Field Distribution. The calculations of the electric field within the cells are based on finite element mesh (11), using a simplified description of the cell morphology (see Fig. 7). In all calculations, the dielectric constant of both the cytoplasm and medium was 80 , their conductance was $0.3 \mathrm{~S} / \mathrm{m}$, the cell diameter was $10 \mu \mathrm{m}$, and the membrane thickness was $3 \mathrm{~nm}$ (with a dielectric constant of 3). The electric field intensity was mapped within the cell, based on the amplitude $(1 \mathrm{~V} / \mathrm{cm})$, frequency $(100 \mathrm{kHz})$ and waveform (sine) of the electric field applied to the cell culture. The force exerted by an inhomogeneous field, such as that created inside the cells on a single tubulin dimer, was calculated based on the direct interaction between the electric field and the dipole. The force exerted on a microscopic polarizable organelle was calculated by the following equation (12):

$$
\langle\vec{F}\rangle=2 \pi r^{3} \epsilon_{\mathrm{m}} \operatorname{Re}[K(\omega)] \vec{\nabla} E_{\mathrm{RMS}}^{2}
$$

where $\langle\vec{F}\rangle$ is the expectation value of the force vector, Re symbolized the real component of the variable, $\vec{\nabla}$ is the divergence of the variable, $\epsilon_{\mathrm{m}}$ is the cytoplasm dielectric constant, $r$ is the tubulin dimer length or particle radius, $E_{\mathrm{RMS}}$ is the RMS value of the electric field, and $K(\omega)$ is the Clausius-Mossotti factor:

$$
\begin{gathered}
K(\omega)=\frac{\epsilon_{p}^{*}-\epsilon_{m}^{*}}{\epsilon_{p}^{*}+2 \epsilon_{m}^{*}} \\
\epsilon^{*}=\epsilon-i \cdot \frac{\sigma}{\psi}
\end{gathered}
$$

where $\epsilon_{\mathrm{p}}, \epsilon_{\mathrm{m}}$ are the complex dielectric constants of the particle and cytoplasm respectively, each of which is calculated from the dielectric constant $(\epsilon)$ and conductance $(\sigma)$ as a function of frequency $(\omega) . K(\omega)$ in this case is always positive at the relatively low frequencies used (i.e., $100 \mathrm{kHz}$ ), assuming that at these frequencies, $\epsilon *_{\mathrm{p}}>\epsilon_{\mathrm{m}}$. This means that the force acting on a polarizable particle will always act in the direction of the convergence of the electric field lines. The terminal velocity of particles due to these forces was calculated using Stoke's law.

In Vivo Experimental Setup. TTField treatment was applied by means of 10-mm-long pairs of parallel, insulated wires (outer diameter, $0.5 \mathrm{~mm}$; insulation thickness, $0.125 \mathrm{~mm}$; Tefzel) placed intradermally on the back of a mouse. Another pair of identical wires was placed parallel to the first pair in each mouse, with an interval of $5 \mathrm{~mm}$ between the pairs. Cell line inoculums were injected $\left(4 \mu \mathrm{l} ; 3 \times 10^{5}\right.$ cells) intradermally in between the two members of each pair of implanted wires. Only one pair was then connected to a voltage amplifier to apply $100 \mathrm{kHz}$ of TTFields treatment to one tumor. The other pair of wires was left disconnected, and the tumor between them served as a paired control of the treated tumor (see Fig. $1 B$ ). Tumors were measured using a caliper. Tumor size was calculated by multiplying maximal tumor length by maximal tumor width. Animal experiments were conducted in accordance with the Technion-Israel Institute of Technology guidelines for the care of laboratory animals.

\section{RESULTS}

Effect of TTFields on Cells in Culture. More than 500 culture dishes were exposed to TTFields. The number of cells in each treatment dish was assessed periodically using colorimetric determination (as described in "Materials and Methods"). Because under control conditions, most of the cell lines had doubling times of less than $24 \mathrm{~h}$ (range, 17-24 h; except for PC-3 for which the doubling time was $73 \mathrm{~h}$ ), treatment duration was at least $24 \mathrm{~h}$. Exposure began $24 \mathrm{~h}$ after seeding and was continued for up to $72 \mathrm{~h}$. In all cell lines tested, 24-h exposure to TTFields at $100 \mathrm{kHz}$ (at an intensity of $1.0-1.4 \mathrm{~V} / \mathrm{cm}$ ) caused significant inhibition of cell proliferation (TER range, $0.14-$ 0.96; $P<0.05$; Fig. $1 C$ ). This effect lasted beyond the exposure time of the cells to TTFields. In fact in some experiments (e.g., malignant melanoma), culture growth was stunted for as long as $72 \mathrm{~h}$ after TTField exposure was terminated (Fig. 2A).

We next checked whether nonreplicating cultures and tissues are affected by TTFields. BHK cultures were maintained in low-serum $(0.1 \%$ FCS $)$ conditions to slow their replication rate. These cultures were then exposed to $100 \mathrm{kHz}$ of TTFields (at an intensity of 1.2 $\mathrm{V} / \mathrm{cm}$ ) for $24 \mathrm{~h}$. No significant difference in cell number between control and TTField-treated cultures was observed under these con- 
A

Fig. 1. Schematic representations of experimental setups in vitro $(A)$ and in vivo $(B)$ are shown. $C$, TTFields inhibit the growth of cancerous cell lines in vitro. Cultures were exposed to $100-\mathrm{kHz}$ TTFields at an intensity of $1-1.4 \mathrm{~V} / \mathrm{cm}$. Ordinate, $T E R$, i.e., the ratio of the decrease in the growth rate of treated cells compared with the growth rate of control cells $\left(\left(\mathrm{GR}_{\mathrm{c}}-\mathrm{GR}_{\mathrm{t}}\right) / \mathrm{GR}_{\mathrm{c}}\right)$. In all four animal cell lines $(\square)$ and seven human cell lines ( $\square$ ) tested, the ratio is greater than 0 , indicating an inhibition in the growth rate of the treated cultures compared with temperature matched controls. All effects were statistically significant $(P<0.05$; Student's $t$ test $)$

C
B
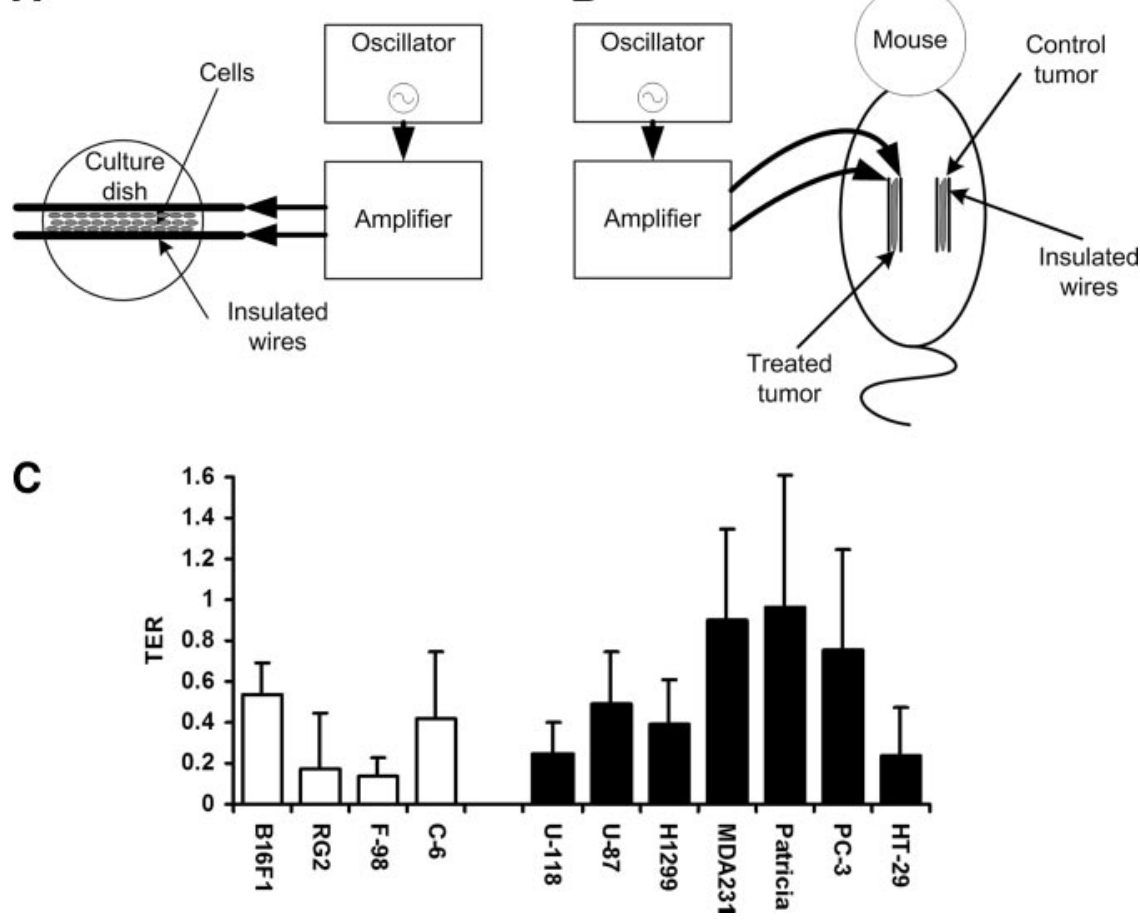

ditions $(P=0.97)$. After returning these cultures to normal media (10\% FCS), normal replication resumed both in cultures exposed to TTFields and in control cultures. We also tested the effect of TTField treatment on the number of viable cells in nonreplicating tissues dissected from rats. Four segments of rat mesentery and four segments of rat diaphragm were exposed to $100 \mathrm{kHz}$ of TTFields at an intensity of $1.2 \mathrm{~V} / \mathrm{cm}$ for $24 \mathrm{~h}$. No differences were observed between the number of viable cells in both types of treated tissues compared with control tissues (mesentery, $P=0.3$; diaphragm, $P=0.54$ ).

To test the relationship between TTField intensity and inhibition of cell proliferation, mouse melanoma (B16F1) and rat glioma (F-98) cell lines were exposed to TTFields of different intensities between 1 and $2.5 \mathrm{~V} / \mathrm{cm}$. The inhibitory effect of TTFields on cell proliferation increased as intensity was raised (Fig. $2 B$ ) until complete proliferation arrest was achieved at intensities of 1.4 and $2.25 \mathrm{~V} / \mathrm{cm}$ in melanoma and glioma cells, respectively.

The effects of TTFields are expected to be frequency dependent in view of the dependence of cell membrane electric impedance on frequency (due to the cell membrane capacitance). These changes in impedance render the fraction of field penetrating the cells a function of frequency. Therefore, we tested the frequency dependence of the inhibitory effect of TTFields on growth rate of cultured melanoma (B16F1) and glioma (F-98) cells. Comparison between the efficacy of the TTFields at different frequencies was performed by normalizing the TER to the electric field intensity. As seen in Fig. $2 C$, the inhibitory effect of TTFields was frequency dependent. Interestingly, the frequency at which maximal inhibition was achieved differed between cell types $(120 \mathrm{kHz}$ versus $\sim 200 \mathrm{kHz}$ for melanoma and glioma, respectively).

The Effects of TTFields on Cellular and Molecular Processes in Proliferating Cells. To gain insight into the cellular processes by means of which TTFields affect cell proliferation, time-lapse microphotography was performed while TTFields were applied to mouse melanoma cultures (see "Materials and Methods"). Several unique processes became evident in time-lapse microphotography of TTField-treated cultures. The most pronounced phenomenon was prolongation of mitosis. In the treated cells, mitosis seemed to begin normally but was prolonged for variable periods of time before completing cleavage into two daughter cells. Fig. $3 A$ shows an exemplary mitosis in a TTFields-treated cell. As seen in the treated cell, mitosis was not complete within $3 \mathrm{~h}$. Due to this proliferation arrest, in treated cultures, mitosis lasted on average $124 \pm 91 \mathrm{~min}$ (mean $\pm \mathrm{SD}, n=53$; range, 40-541 $\mathrm{min}$ ), whereas under control conditions, average mitosis duration was $62 \pm 8 \mathrm{~min}$ from cell rounding to cytokinesis (mean $\pm \mathrm{SD}, n=12$; range, 47-78 min). This prolongation is statistically significant $(P<0.01$, Mann-Whitney $U$ test).

The second major phenomenon, seen in the TTField-treated melanoma cultures, was that one-fourth of cells undergoing mitosis were destroyed as the formation of the cleavage furrow approached complete cell separation. During this process, the cell membrane ruptured, and many small membrane blebs formed, resembling post-mitotic apoptotic cell death (13). Two exemplary cells undergoing such destruction are shown in Fig. 3, $B$ and $C$. Destructive effects were observed only in mitotic cells, whereas quiescent cells remained morphologically and functionally intact.

The third phenomenon, seen only in TTField-treated cultures, was nuclear rotation. In early mitosis, after cell rounding, nuclei could be seen rotating within the cell. A full rotation lasted on average $15 \mathrm{~min}$. This effect resembles the whole-cell rotation previously described during exposure to intermediate-frequency alternating electric fields $(7,8)$.

A fundamental characteristic of electric fields is that at any point in space, they have a defined orientation corresponding to the direction of the force they exert on charges and polar elements. With regard to the latter, the force exerted by the field is maximal when the dipole is oriented in the direction of the field. With regard to the above, there are two main structural differences between quiescent and dividing cells. One is that the latter contain highly polar, spatially oriented microtubules and that they develop a directional, hourglass-shaped cell morphology during the cytokinesis phase. In view of these facts, one may expect that the electric field forces will have maximal effect 
Fig. 2. Time, field frequency, and intensity dependence of the effect of TTFields on malignant melanoma (B16F1, left column) and glioma cell (F-98, right column) proliferation. A, the number of cells in untreated cultures (control; $\square$ ) as compared with cultures treated with TTFields ( $\square$ ). The number of cells at each time point $\left(O D_{i}\right)$ was normalized by the number of cells in the culture before initiation of treatment $\left(O D_{0}\right)$. The number of control cells is seen to roughly double every $24 \mathrm{~h}$ throughout the experiment. TTFields were applied for $24 \mathrm{~h}$ continuously (solid lines) at $100 \mathrm{kHz}$ in the melanoma cultures and at 200 $\mathrm{kHz}$ in the glioma cultures. The increase in the number of treated melanoma (left) and glioma (right) cells over time is significantly smaller than control cells $(P<0.001)$. B, the effect of 24-h exposure to TTFields of increasing intensities. The magnitude of the effect is expressed using the TER. The inhibitory effect of the TTFields on proliferation increases with intensity in both cell types. Complete proliferation arrest $($ TER $=1)$ is seen at 1.35 and $2.25 \mathrm{~V} / \mathrm{cm}$ in melanoma and glioma cells, respectively. $E F$, electric field. $C$, change in the melanoma (left) and glioma (right) growth rate after $24 \mathrm{~h}$ of exposure to TTFields of different frequencies is normalized to the field intensity $(T E R / E F)$. A window effect is seen with maximal inhibition by TTFields at $120 \mathrm{kHz}$ in melanoma cells and at $\sim 200 \mathrm{kHz}$ in glioma cells. Data are mean + SE.

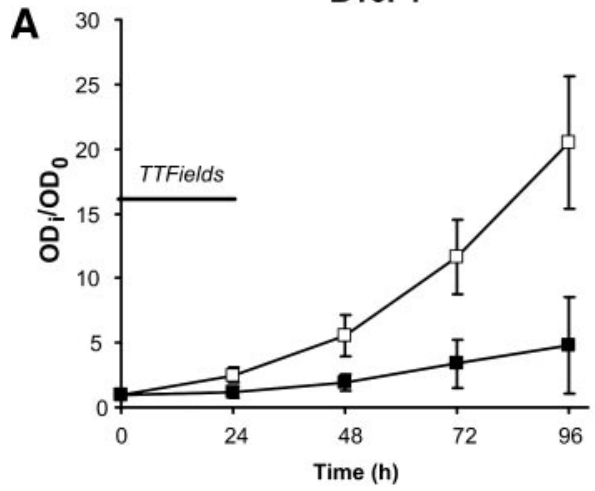

B

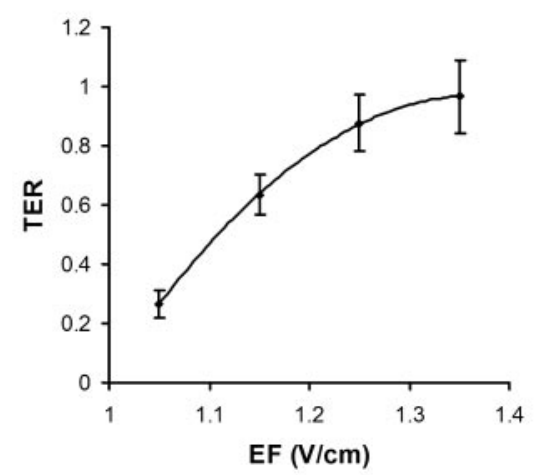

C

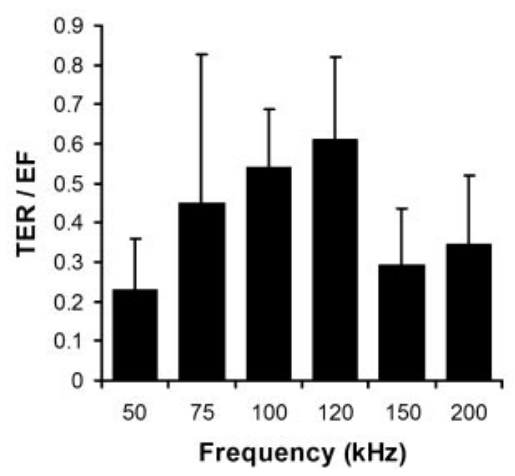

F-98
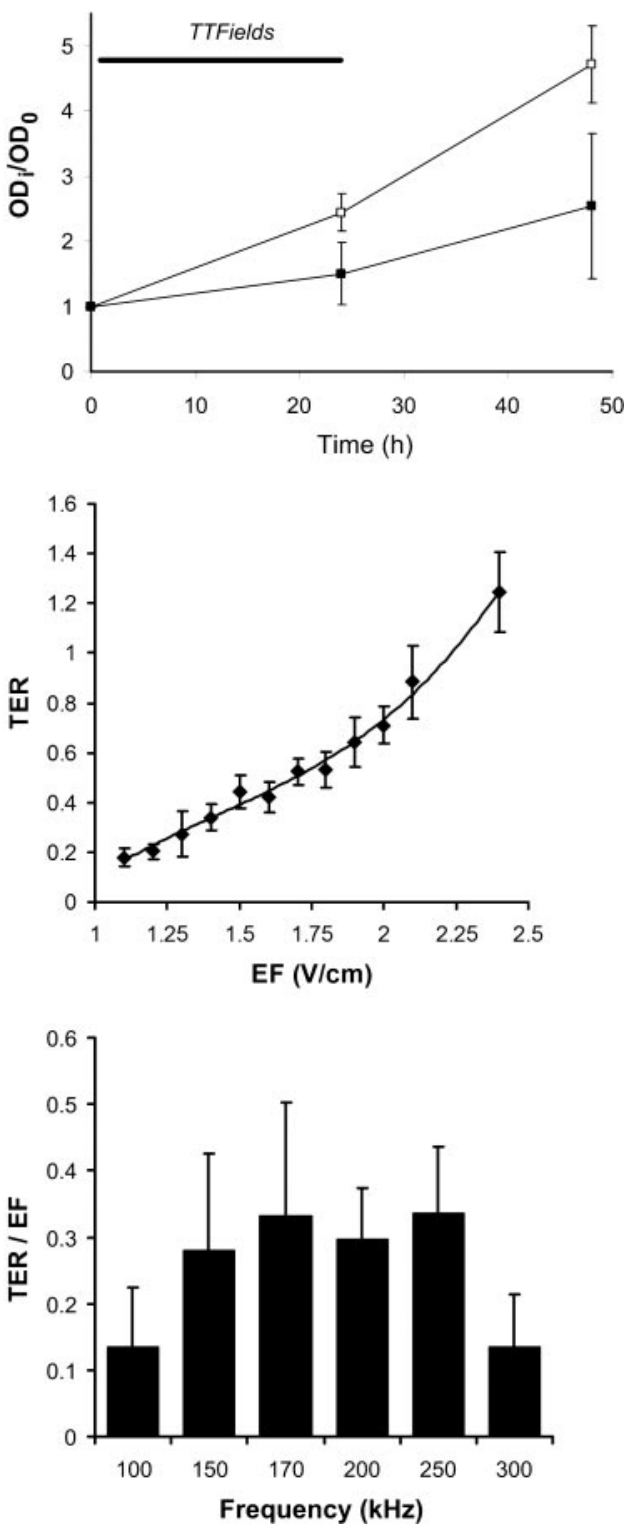

on the mitotic process when it is oriented along the lines of force of the field. To investigate this point, we fixed melanoma cell cultures and stained them with toluidine blue, immediately after $24 \mathrm{~h}$ of TTField treatment, to demonstrate mitoses and to distinguish vital from damaged or dead cells. The live and damaged mitotic cells (at the time of fixation) were grouped according to the orientation of their cleavage axis relative to the electric field direction. The cells were counted separately in each of four equal sectors that form angles of $0^{\circ}$, $45^{\circ}$ (two sectors, 45 and 135 ), and $90^{\circ}$ relative to the field direction. As seen in Fig. 4A, the live cells were randomly distributed in all sectors. In contrast, a much higher proportion of the damaged cells had their axis of division oriented along the field: $56 \%$ at $0^{\circ}$ versus an average of $15 \%$ in each of the other orientations. Surprisingly, the number of cells per unit area in the two $45^{\circ}$ sectors was found to be one-half that in the $0^{\circ}$ sector. This finding may serve as an indication of an additional effect of TTFields: orientation of the cell division in the field direction. The cells in each of the above spatially oriented defined groups were further divided according to stages of mitosis at the time of fixation. At all stages, a higher fraction of damaged cells had their axis of division oriented along the field. Moreover, 74\% of the parallel oriented cells were damaged while being in metaphase (Fig. 4B).

The spatially organized mitotic spindle, which forms in dividing cells, consists of microtubules that have very large electric dipole moments (14) and may therefore be disoriented by the forces of the electric fields $(15,16)$. Actin filaments are also polar, however, they have no defined spatial orientation within the cells and are therefore not expected to be significantly affected by the fields. This prompted us to test whether TTFields disrupt mitosis by interfering with the normal formation, orientation, and movement of microtubules as compared with actin filaments as follows: Melanoma cell cultures were treated with TTFields for $24 \mathrm{~h}$. After treatment, the cells were fixated, stained with monoclonal antibodies directed against microtubules and actin filaments, as well as for DNA, and thereafter studied with fluorescence microscopy (see "Materials and Methods"). In control cultures, $95 \%$ of cells undergoing mitosis exhibited the normal stages of mitosis with intact mitotic spindles. However, in TTFieldtreated cultures, more than one-half of the mitoses were abnormal. 
$+2 \mathrm{~h}$

A
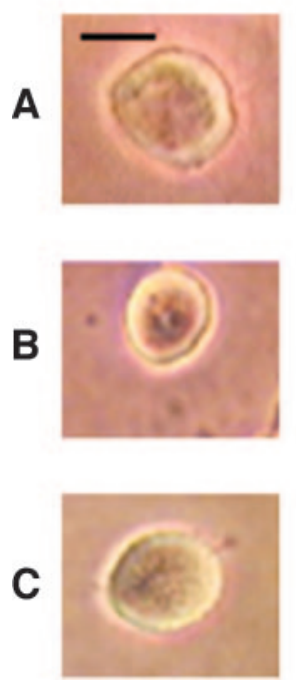

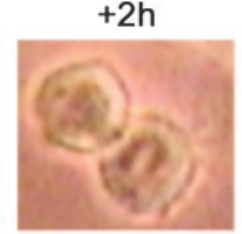

$+30 \mathrm{~m}$

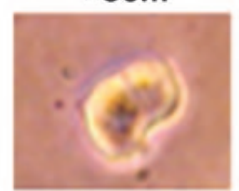

$+30 \mathrm{~m}$

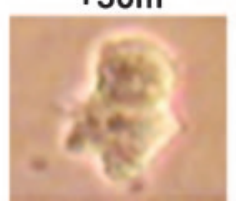

$+3 h$

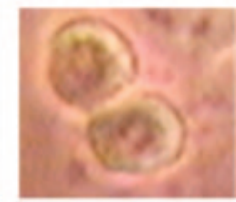

$+50 \mathrm{~m}$

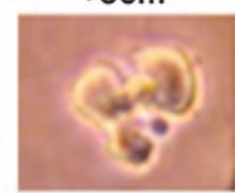

$+60 \mathrm{~m}$

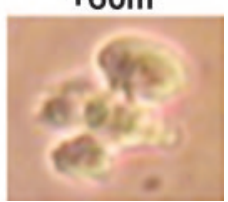

Fig. 3. Time-lapse microphotography of malignant melanoma cells exposed to TTFields. A, an example of a cell in mitosis arrested by TTFields. Contrary to normal mitosis, the duration of which is less than $1 \mathrm{~h}$, the depicted cell is seen to be stationary at mid-cytokinesis for $3 \mathrm{~h} . B$ and $C$, two examples of disintegration of TTFields-treated cells during cytokinesis. Three consecutive stages are shown: cell rounding (left); formation of the cleavage furrow (middle); and cell disintegration (right). Scale bar $=10 \mu \mathrm{m}$.

Fig. 5 shows examples of the different forms of abnormal mitosis seen under TTField treatment. These included polypoid cells in prophase, ill-separated, multi-spindled and single-spindled cells in metaphase, asymmetric anaphases, and a large proportion of cells in metaphase $(>20 \%)$ with rosette shaped chromosome assemblies. The normal and abnormal stages of mitosis in control and TTField-treated cultures are summarized and compared in Fig. 5G. In general, these abnormalities may serve as an indication of interference of TTFields with the normal behavior of the microtubules. In contrast, staining for actin filaments showed no difference between TTField-treated and control cultures.

Effect of TTFields on Tumors in Vivo. To test whether TTFields are effective in destroying tumor cells in vivo, we tested their effect on two animal tumor models: C57BL/6 mice inoculated intradermally with malignant melanoma cells (B16F1) and BALB/c mice inoculated intradermally with adenocarcinoma cells (CT-26). TTFields were generated between implanted (intradermal) wholly insulated wires placed on both sides of the tumor (see Fig. $1 B$ ). Mice with implanted electrodes were treated for 3-6 days continuously beginning 1 day after cell line inoculation. We found that $100-200 \mathrm{kHz}$ of TTFields at low intensities of $<2 \mathrm{~V} / \mathrm{cm}$ effectively inhibited malignant melanoma growth compared with the growth of nontreated control tumors. Photographs of examples of treated and nontreated malignant melanoma tumors are given in Fig. 6 for comparison. Treated tumors were significantly smaller than control tumors at the end of treatment (average treated tumor size was $47 \%$ of control tumor size; $n=78$ mice, $P<0.001$; Student's $t$ test). Histopathological analysis of treated tumors showed extensive necrosis with aggregations of kariorrhectic and kariolytic debris (Fig. 6F). To test whether TTFields are effective on different tumor types, BALB/c mice with intradermal adenocarcinomas were treated with the same field parameters. Photographs of examples of such a treated and a nontreated adenocarcinoma tumors are provided for comparison in Fig. $6 \mathrm{~B}$. The average effect of TTFields on adenocarcinoma carrying mice was less dramatic than that seen for malignant melanoma (average treated tumor size was $73 \%$ of control tumor size at the end of treatment; $n=14$ mice). After treatment, the tumors and their adjacent tissues were fixated, stained with $\mathrm{H} \& \mathrm{E}$, and analyzed histopathologically. No damage to the surrounding tissues was detected.

\section{DISCUSSION}

In this study, we have shown that when properly tuned, very low-intensity, intermediate-frequency electric fields (TTFields) stunt the growth of cancerous cells. We have demonstrated this inhibitory effect in all proliferating cell types tested, whereas, nonproliferating cells and tissues were unaffected. Interestingly, different types of cancerous cells showed specific intensity and frequency dependences of TTField inhibition. We have demonstrated that two main processes occur at the cellular level during exposure to TTFields: arrest of proliferation and cell destruction. The damage caused by TTFields to these replicating cells was shown to be dependent on the orientation of the division process in relation to the field vectors, indicating that this effect is nonthermal. Indeed, temperature measurements made within culture dishes during treatment and on the skin above treated tumors in vivo, showed no significant elevation in temperature compared with control cultures/mice. Also, TTFields caused the dividing cells to orient in the direction of the applied field in a manner similar to that described in cultured human corneal epithelial cells exposed to constant electric fields (17). At the subcellular level, we have found evidence indicating that TTFields disrupt the normal polymerizationdepolymerization process of microtubules during mitosis. Indeed, the described abnormal mitotic configurations seen after exposure to

A

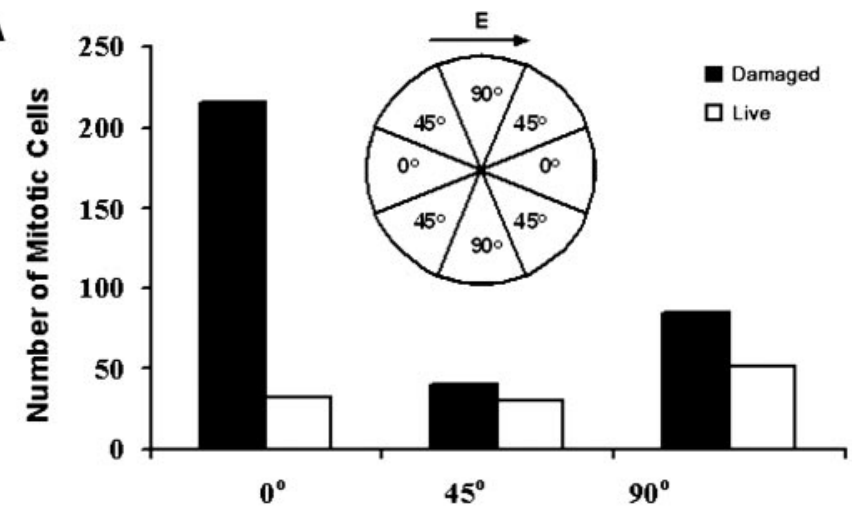

B

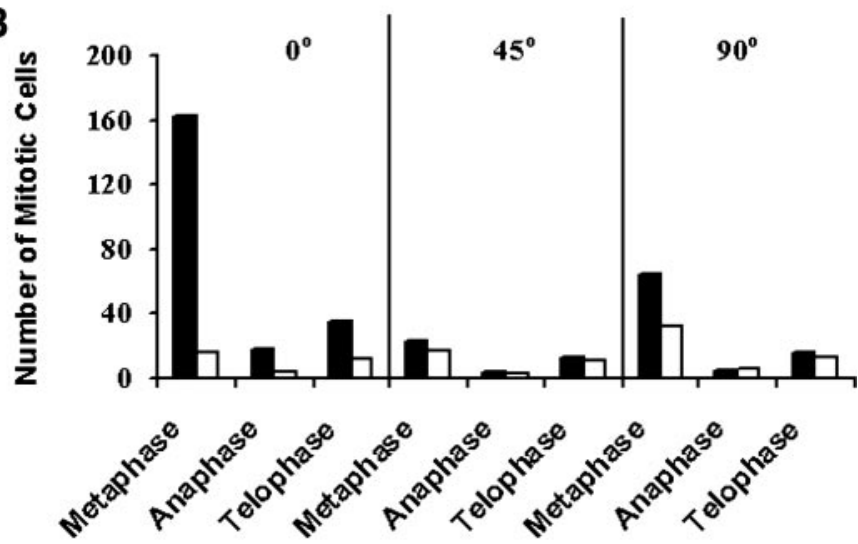

Fig. 4. Dependency of TTFields-induced cellular damage on the orientation axis of cell division relative to field direction. Ordinate represents the number of mitotic cells counted in four TTField-treated malignant melanoma cultures $(100 \mathrm{kHz})$. A, total number of

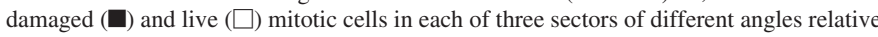
to the field direction (inset). The number of damaged cells is more than 5-fold larger than the corresponding number of live cells when division is aligned at or close to $0^{\circ}$ relative to the electric field direction. In sectors of other angles, the number of damaged cells only slightly exceeds the live ones. Note that because the $45^{\circ}$ area is double that of each of the other two sectors, the number of cells presented in this orientation was halved. $B$, dividing cell sensitivity to fields of different orientation at different stages of mitosis. When cell division axis is aligned at $0^{\circ}$ to the electric field, the number of damaged cells ( $\square$ ) is significantly larger than that of intact cells $(\square)$ at all three phases of mitosis. However, the highest number of damaged cells in this orientation is seen at metaphase (8-fold more than intact cells). 

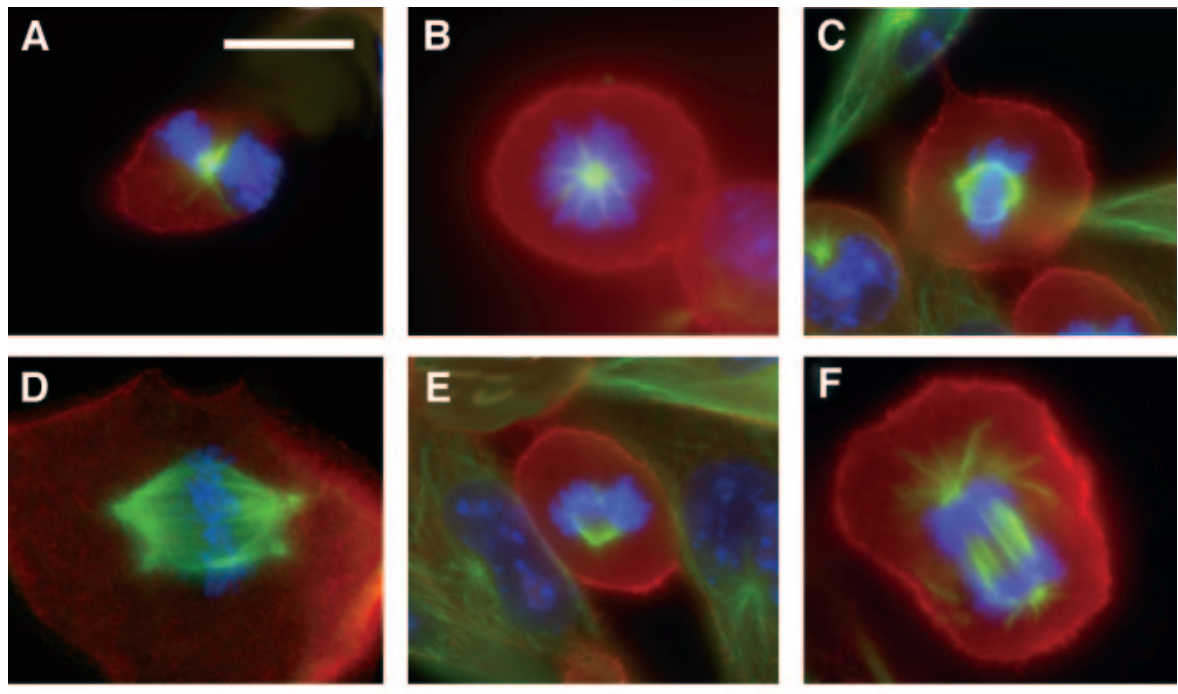

Fig. 5. Immunohistochemical staining of abnormal mitotic figures in TTFields-treated cultures. Malignant melanoma cultures $(n=4)$ were treated for $24 \mathrm{~h}$ at $100 \mathrm{kHz}$ and then stained with monoclonal antibodies for microtubules (green), actin (red), and DNA (blue). The photomicrographs show exemplary abnormal mitoses including: polyploid prophase $(A)$; rosette $(B)$; ill separated metaphase $(C)$; multispindled metaphase $(D)$; single-spindled metaphase $(E)$; and asymmetric anaphase $(F)$. $G$, the percentage of treated $(\square)$ and control ( $\square)$ mitotic cells in each of the normal and abnormal phases of mitosis.

G

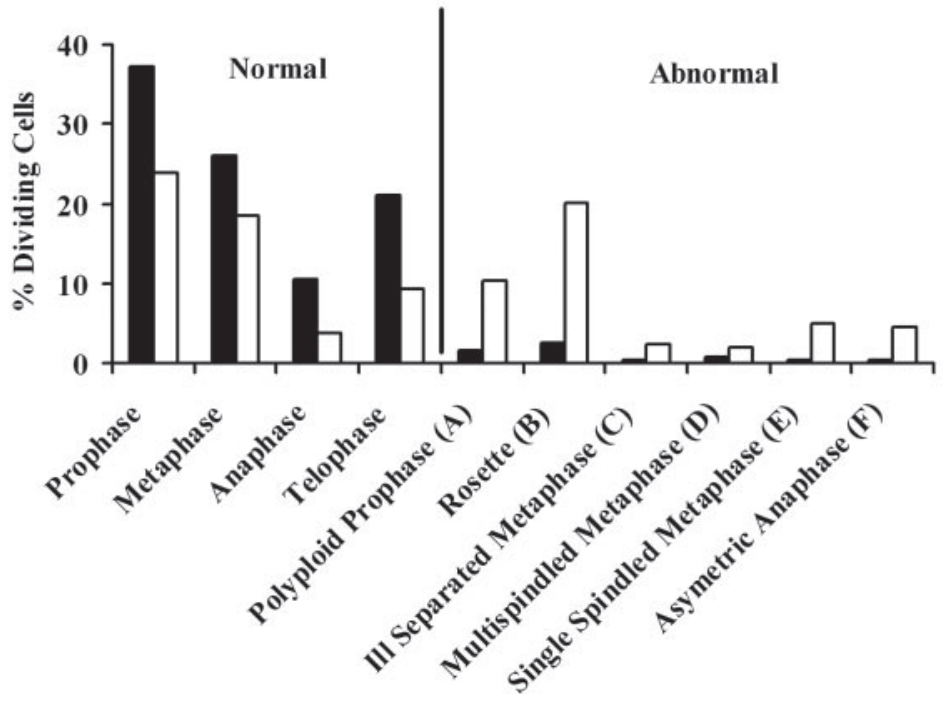

TTFields are similar to the morphological abnormalities seen in cells treated with agents that interfere directly $(18,19)$ or indirectly $(20-$ 22) with microtubule polymerization (e.g., Taxol).

To explain how TTFields cause orientation-dependent damage to dividing cancerous cells and disrupt the proper formation of the mitotic spindle, we modeled the forces exerted by TTFields on intracellular charges and polar particles using finite element simulations (see "Materials and Methods"). We identified two main mechanisms by means of which the electric fields may affect dividing cells. The first relates to the field effect on polar macromolecule orientation. Within this framework, during the early phases of mitosis, i.e., in pre-telophase, when tubulin polymerization-depolymerization drives the proliferation process, the electric field forces any tubulin dimers, positioned further than $14 \mathrm{~nm}$ away from the growing end of a microtubule, to orient in the direction of the field (Fig. 7A). This force moment, $\left(10^{-5} \mathrm{pN}\right)$ acting on the dimers, is sufficient to interfere with the proper process of assembly and disassembly of microtubules that is essential for chromosome alignment and separation (23). This effect can explain the mitotic arrest of TTField-treated cells (24). The second mechanism, which interferes with cell division and is most likely to play an important role in cell destruction, becomes dominant during cleavage. As seen in the simulations depicted in Fig. $7 B$, the electric field within quiescent cells is homogenous, whereas the field inside mitotic cells, during cytokinesis, is not homogenous. We see an increased field line concentration (indicating increased field intensity) at the furrow, a phenomenon that highly resembles the focusing of a light beam by a lens. This inhomogeneity in field intensity exerts a unidirectional electric force on all intracellular charged and polar entities, pulling them toward the furrow (regardless of field polarity). For example, for a cleavage furrow that reached a diameter of $1 \mu \mathrm{m}$ in an external field of only $1 \mathrm{~V} / \mathrm{cm}$, the force exerted on the microtubules is in the order of $5 \mathrm{pN}$. This magnitude is compatible with the reported forces necessary to stall microtubule polymerization that is $4.3 \mathrm{pN}$ (25). With regard to other particles such as cytoplasmatic organelles, they are polarized by the field within dividing cells. Once polarized, the forces acting on such particles may reach values up to an order of $60 \mathrm{pN}$ resulting in their movement toward the furrow at velocities that may approach $0.03 \mu \mathrm{m} / \mathrm{s}$. At such velocity, cytoplasmatic organelles would pile up at the cleavage furrow within a few minutes, interfering with cytokinesis and possibly leading to cell destruction. We also found that the electric forces acting on intracellular particles are maximal when the axis of division is aligned with the external field. This is consistent with the dependence of the destructive effect of TTFields on the angle between division axis and the field (Fig. 4). In addition, the calculated dependence of the magnitude of this force on frequency (data not shown) is consistent with the experimentally determined frequency dependence of the 
Fig. 6. In vivo effects of TTFields on intradermal tumors in mice. Malignant melanoma $(A)$ and adenocarcinoma $(B)$ tumor cells were injected in two parallel locations intradermally on the back of each mouse. Only the tumor on the left side of the mouse was treated. After 4 days of TTFields treatment (at $100 \mathrm{kHz}$ ), no tumor can be discerned on the treated side, whereas on the untreated side a large tumor has grown. $C-F$, histological sections of TTFields-treated intradermal melanoma versus a control (untreated) melanoma on the same mouse. $C$, after $\mathrm{H} \& \mathrm{E}$ staining, a large $(5 \mathrm{~mm}$ diameter) nodule of melanoma cells can be seen in the dermis of the control tumor $(\times 40)$. Note that due to the large size of the tumor, its deep portion has been lost in preparation. $D$, treated tumor; only two small $(<0.4 \mathrm{~mm}$ diameter $)$ nodules are present (scale bar $=0.5 \mathrm{~mm}$ ). The nontumor structures of the dermis are morphologically intact. $E$, control tumor, malignant melanoma cells appear intact and viable $(\times 200)$. $($ Scale bar $=100 \mu \mathrm{m}) . F$, only necrotic tissue and cellular debris are seen in the treated tumor.
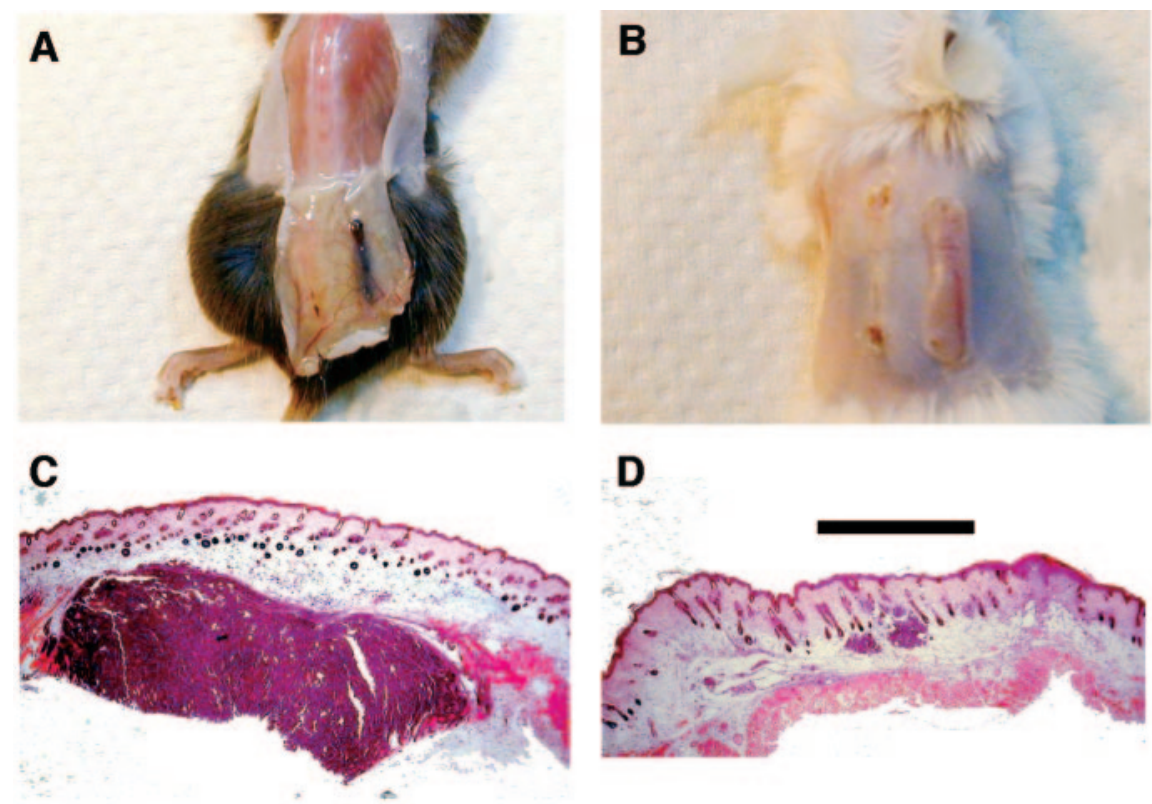

D
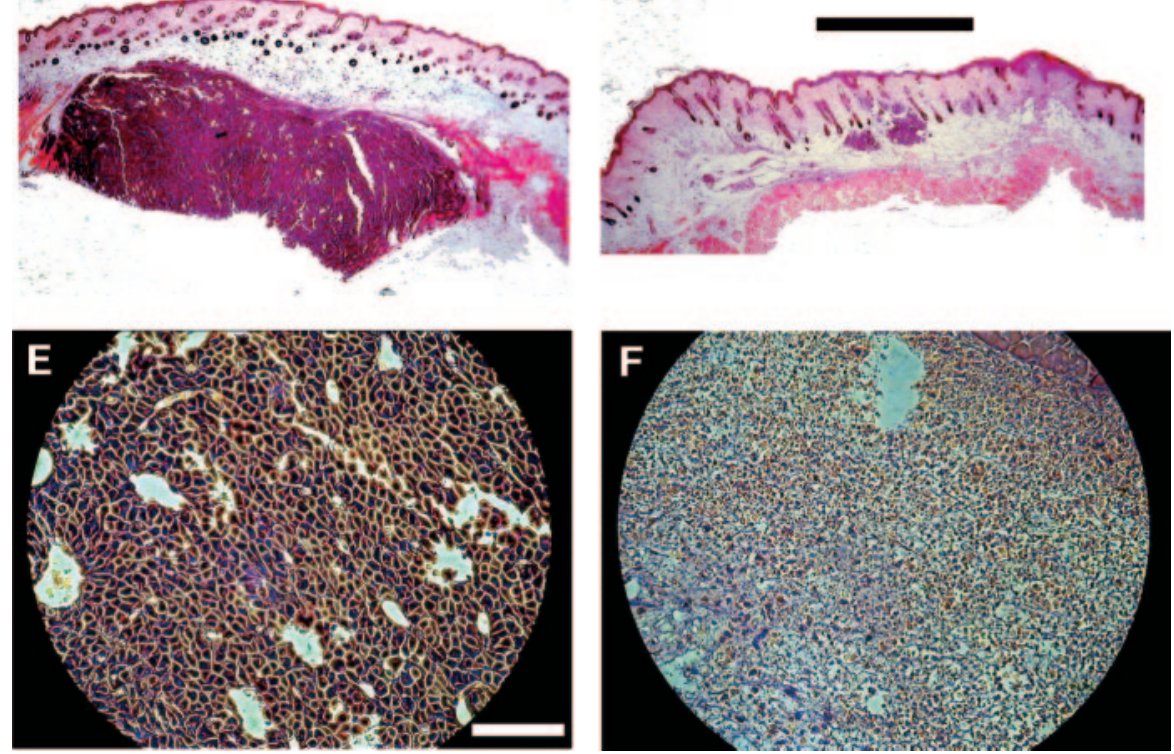

A

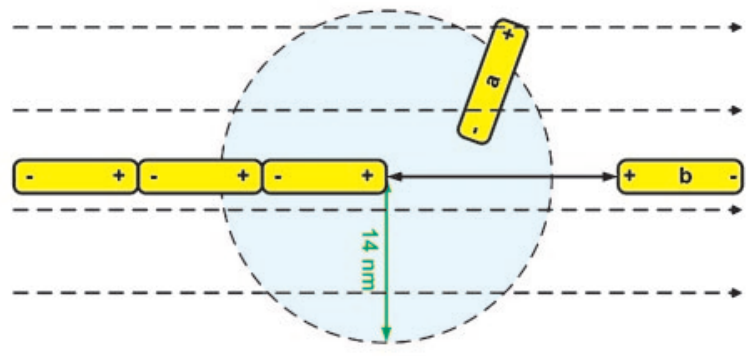

B
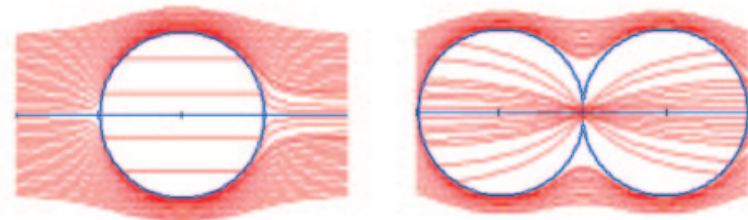

Fig. 7. A, schematic representation of two tubulin dimers positioned near the tip of an elongating microtubule in a dividing cell. The force that a $1-\mathrm{V} / \mathrm{cm}$ extracellular TTField exerts on a tubulin dimer located less than $14 \mathrm{~nm}$ away from the microtubule $(a)$ is smaller than the force exerted by the polar microtubule tip, and therefore it will align according to the field generated by the microtubule. In contrast, dimers further than $14 \mathrm{~nm}$ from the end of the microtubule $(b)$ are aligned by the forces of the TTFields (dashed lines) in a direction that may not be compatible with the polymerization-depolymerization process. $B$, finite element mesh simulation of the lines of force of the electric field inside a quiescent cell (left) and a cell undergoing mitotic cytokinesis (right). The diameters of the cells in the simulations was 10 $\mu \mathrm{m}$ and membrane thickness $3 \mathrm{~nm}$. Inside the quiescent cell, the electric field is mostly uniform (equal distances between the lines of force). In contrast, in the dividing cell, the field is inhomogenous - the field intensity (line density) increases toward the cleavage furrow. inhibitory effect of TTFields on melanoma and glioma cell proliferation (Fig. 2C).

In conclusion, we have demonstrated that TTFields inhibit both the proliferation of malignant cells in culture and the growth of tumors in mice while showing no general side effects or local histopathological damage. The mechanism of action of the fields is, at least in part, dependent on disruption of the microtubules of the mitotic spindle and the electric forces resulting from focusing of the field in the dividing cells. The highly specific effects of these fields on dividing cells, together with the relative ease of applying them, focusing them, and screening from them, make them an attractive candidate to serve as a novel treatment modality for cancer.

\section{REFERENCES}

1. Polk C. Therapeutic applications of low-frequency sinusoidal and pulsed electric and magnetic fields. In: Bronzino JD, editor. The biomedical engineering handbook. Boca Raton, FL: CRC Press, Inc.; 1995. p. 1404-16.

2. Palti Y. Stimulation of internal organs by means of externally applied electrodes J Appl Physiol 1966;21:1619-23.

3. Basset CA. The development and application of pulsed electromagnetic fields (PEMFs) for ununited fractures and arthrodeses. Clin Plast Surg 1985;12:259-77.

4. Elson E. Biologic effects of radiofrequency and microwave fields: in vivo and in vitro experimental results. In: Bronzino JD, editor. The biomedical engineering handbook. Boca Raton, FL: CRC Press, Inc.; 1995. p. 1417-23.

5. Chou CK. Radiofrequency hyperthermia in cancer therapy. In: Bronzino JD, editor. The biomedical engineering handbook. Boca Raton, FL: CRC Press, Inc.; 1995. p. 1424-30.

6. Takashima S, Schwan HP. Alignment of microscopic particles in electric fields and its biological implications. Biophys J 1985;47:513-8.

7. Zimmermann U, Vienken J, Pilwat G. Rotation of cells in an alternating electric field: the occurrence of a resonance frequency. Z Naturforsch C 1981;36:173-7.

8. Holzapfel C, Vienken J, Zimmermann U. Rotation of cells in an alternating electric field: theory and experimental proof. J Membr Biol 1982;67:13-26. 
9. Pawlowski P, Szutowicz I, Marszalek P, Fikus M. Bioelectrorheological model of the cell. 5. Electrodestruction of cellular membrane in alternating electric field. Biophys J 1993;65:541-9.

10. Jost LM, Kirkwood JM, Whiteside TL. Improved short- and long-term XTT-based colorimetric cellular cytotoxicity assay for melanoma and other tumor cells. J Immunol Methods 1992;147:153-65.

11. Volakis JL, Chatterjee A, Kempel LC. Finite element method electromagnetics: antennas, microwave circuits, and scattering applications. New York, NY: IEEE/ OUP; 2001.

12. Pohl AH. Dielectrophoresis. Cambridge, UK: Cambridge University Press; 1978.

13. Endlich B, Radford IR, Forrester HB, Dewey WC. Computerized video time-lapse microscopy studies of ionizing radiation-induced rapid-interphase and mitosis-related apoptosis in lymphoid cells. Radiat Res 2000;153:36-48.

14. Alberts B, Roberts K, Lewis J, Raff M, Watson JD. Molecular biology of the cell. 2nd ed. New York: Garland Publishing, Inc.; 1989. p. 1216.

15. Meggs WJ. Electric fields determine the spatial organization of microtubules and actin filaments. Med Hypotheses 1988;26:165-70.

16. Cho MR, Thatte HS, Lee RC, Golan DE. Reorganization of microfilament structure induced by ac electric fields. FASEB J 1996;10:1552-8.

17. Zhao M, Forrester JV, McCaig CD. A small, physiological electric field orients cell division. Proc Natl Acad Sci USA 1999;96:4942-6.
18. Jordan MA, Thrower D, Wilson L. Effects of vinblastine, podophyllotoxin and nocodazole on mitotic spindles: implications for the role of microtubule dynamics in mitosis. J Cell Sci 1992;102:401-16.

19. Rowinsky EK, Donehower RC. Paclitaxel (Taxol). N Engl J Med 1995;332: $1004-14$.

20. Kline-Smith SL, Walczak CE. The microtubule-destabilizing kinesin XKCM1 regulates microtubule dynamic instability in cells. Mol Biol Cell 2002;13:2718-31.

21. Kapoor TM, Mayer TU, Coughlin ML, Mitchison TJ. Probing spindle assembly mechanisms with monastrol, a small molecule inhibitor of the mitotic kinesin, Eg5. J Cell Biol 2000;150:975-88.

22. Maiato H, Sampaio P, Lemos CL, et al. MAST/Orbit has a role in microtubulekinetochore attachment and is essential for chromosome alignment and maintenance of spindle bipolarity. J Cell Biol 2002;157:749-60.

23. Gagliardi LJ. Electrostatic force in prometaphase, metaphase, and anaphase-A chromosome motions. Phys Rev E Stat Nonlin Soft Matter Phys 2002;66:011901.

24. Fishkind DJ, Silverman JD, Wang YL. Function of spindle microtubules in directing cortical movement and actin filament organization in dividing cultured cells. J Cell Sci 1996;109:2041-51.

25. Dogterom M, Yurke B. Measurement of the force-velocity relation for growing microtubules. Science 1997;278:856-60. 